\title{
Ambiente Virtual Imersivo para ensino em Redes de Computadores: uma proposta usando Agentes Inteligentes
}

\author{
Fabrício Herpich ${ }^{1}$, Felipe Becker Nunes ${ }^{2}$, Gleizer Bierhalz Voss ${ }^{3}$, Rafaela Ribeiro \\ Jardim $^{1}$ e Roseclea Duarte Medina ${ }^{1}$ \\ ${ }^{1}$ PPGI - Universidade Federal de Santa Maria (UFSM) \\ ${ }^{2}$ PPGIE - Universidade Federal do Rio Grande do Sul (UFRGS) \\ ${ }^{3}$ Instituto Federal Farroupilha (IF Farroupilha) \\ \{fabricio.herpich, nunesfb, gleizer.voss, rafa.rjardim, \\ roseclea.medina\} @gmail.com
}

\begin{abstract}
This paper presents the development of an intelligent agent ELAI, through the use of the Non Player Character (NPC) existing strategy in the virtual world OpenSim. It provides support for teaching Computer Networks $(C N)$, being context-awaraness in regards to the level of experience (expertise) of students. In order to maximize the flexibility of the interactions between the student, through his avatar and the NPC, an interconnection of this with a chatterbot was established, whose knowledge base was adapted to the context of $C N$.
\end{abstract}

Resumo. Este trabalho apresenta o desenvolvimento do agente inteligente ELAI, por meio do uso da estratégia de Non Player Character (NPC) existente no mundo virtual OpenSim. Ele provê suporte para o ensino de Redes de Computadores (RC), sendo sensivvel ao contexto no que diz respeito ao nível de experiência (expertise) dos estudantes. De forma a maximizar a flexibilidade das interações entre o estudante, por meio de seu avatar e o NPC, foi estabelecida uma interconexão deste com um chatterbot, cuja base de conhecimento foi adaptada ao contexto de RC.

\section{Introdução}

Com o crescente uso das Tecnologias de Informação e Comunicação (TICs) no âmbito educacional, diversas necessidades foram surgindo e alterando os cenários atuais, fazendo-se necessária a reflexão sobre novos paradigmas da computação na educação. Neste contexto, muitas pesquisas vêm sendo realizadas em torno de ambientes virtuais imersivos, que são capazes de proporcionar ao estudante interação com objetos de aprendizagem e a capacidade de estar imerso no ambiente, como em Silva et al. (2011) e Piovesan et al. (2013).

A criação de ambientes imersivos voltados à educação necessita que diversos fatores sejam considerados, e.g., objetivos pedagógicos e estratégias de ensino bem definidas com base em teorias de aprendizagem, design amigável e objetos capazes de incentivar a interação e colaboração entre os usuários. Pois, segundo Medina (2004), a aprendizagem obtida através das experiências pessoais dos participantes e das suas interações com outros participantes, tornam-se mais produtivas, consolidadas $\mathrm{e}$ dinâmicas. 
Neste sentido, outro aspecto relacionado ao desenvolvimento destes ambientes refere-se do suporte oferecido por agentes inteligentes, pois conforme Russel e Norvig (1995) os agentes devem ter a capacidade de perceber o ambiente ao qual estão inseridos e, através de sensores, atuarem adotando as melhores ações possíveis para resolverem uma situação. A relevância de agentes inteligentes voltados à educação também é discutida por Soliman e Guetl (2010), no qual os autores afirmam que em ambientes virtuais de aprendizagem os estudantes têm uma grande flexibilidade, diante de inúmeras oportunidades de aprendizagem e, para tanto, faz-se necessário um apoio e uma orientação inteligente.

O apoio inteligente proporcionado por agentes em ambientes de aprendizagem pode contribuir significativamente no processo de ensino-aprendizagem dos estudantes, pois ainda conforme Soliman e Guetl (2010), o agente pode atuar como professor, facilitador da aprendizagem ou até mesmo como um colega em ambientes colaborativos. $\mathrm{Ou}$ seja, orientando o estudante no ambiente virtual, explicando tópicos, fazendo perguntas, dando feedback, ajudando o aluno a colaborar com os outros estudantes, dando apoio à aprendizagem personalizada e agindo sobre o aluno em diferentes momentos e lugares.

O presente trabalho tem como objetivo principal o desenvolvimento de um agente inteligente com múltiplas habilidades e características, implementado dentro de um mundo virtual (MV) denominado $\mathrm{TCN}^{5}$ de Voss (2014), através de NPCs. O agente inteligente foi denominado como ELAI, acrônimo para Intelligent Agent adaptable to the Level of Expertise from Students, da sigla em português Agente Inteligente adaptável ao Nível de Expertise dos Estudantes, que é sensível ao contexto do nível de expertise do educando, visando o ensino de Redes de Computadores.

\section{Desenvolvimento da Proposta e Resultados Preliminares}

Este estudo apresenta um projeto de pesquisa em desenvolvimento, que envolve a implantação do agente inteligente ELAI, que foi estruturado para efetuar a análise do nível de experiência do estudante e adaptar suas ações conforme as preferências individuais do educando. Para tanto, o agente foi implementado junto ao ambiente virtual imersivo $\mathrm{TCN}^{5}$, desenvolvido na plataforma OpenSim e que tem como objetivo o ensino de Redes de Computadores.

O agente inteligente ELAI foi estruturado por meio da inserção de scripts nativos do OpenSim, denominados de OpenSimulator Scripting Language (OSSL), no qual foram acopladas múltiplas habilidades considerando os critérios elencados por Franklin e Graesser (1996) para agentes inteligentes, e.g. interação, autonomia, reatividade, próatividade, atividade contínua, adaptabilidade, mobilidade e personalidade (Figura 1).

Para atender aos requisitos elencados anteriormente, foi implementado e incorporado ao agente inteligente ELAI um sensor de movimento que é ativado quando outros avatares (representação dos usuários) entram no raio de ação monitorado pelo agente. O uso do sensor possibilita que as habilidades do ELAI sejam aprimoradas, provendo dessa forma os seguintes recursos: percepção do ambiente; atuadores sobre o próprio ambiente ou seus usuários; autonomia para realizar determinadas atividades; reatividade ao perceber o ambiente e responder às suas mudanças; pró-atividade ao 
tomar iniciativa; mobilidade relacionada a capacidade de se mover; e de atividade contínua sempre estando em processo de atividade.

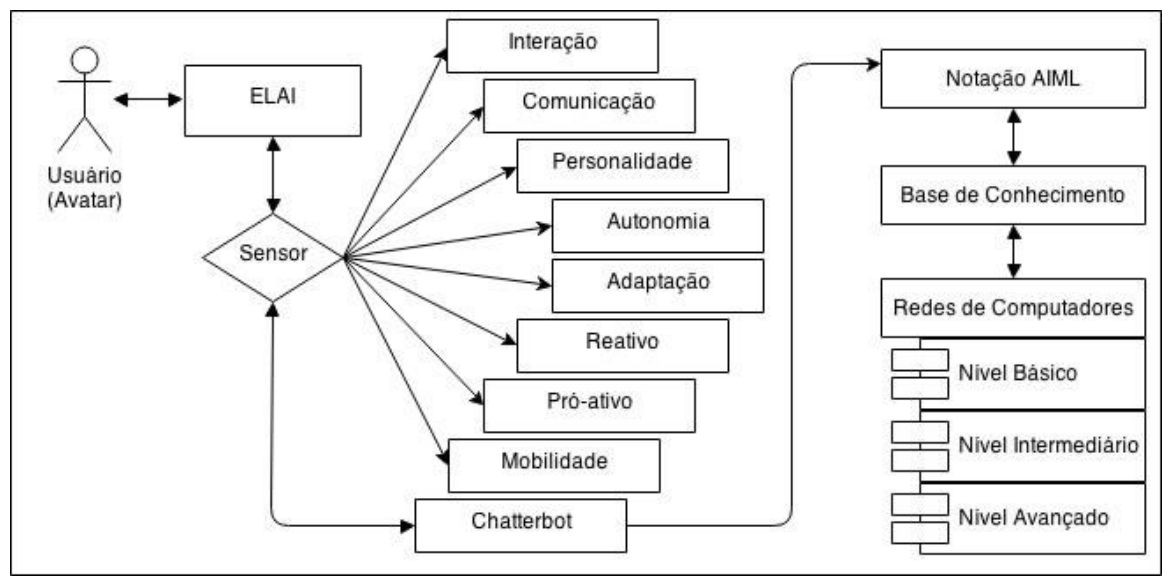

Figura 1. Arquitetura do Agente Inteligente ELAI

Os requisitos de mobilidade e reatividade do agente se revelam quando o estudante se aproxima e ingressa no raio de ação do ELAI, o qual executa a reação de movimentar-se ao encontro da posição do estudante. Além disso, caso o estudante se movimente dentro desse raio de ação, o ELAI irá segui-lo, de forma a proporcionar uma sensação de assistência e monitoramento, sendo possível lhe oferecer ajuda.

No mesmo momento que o agente identifica a proximidade do estudante em seu raio de ação, uma mensagem de boas-vindas é emitida, estabelecendo um canal de comunicação por meio do chat para proporcionar uma interação usuário/agente. Outro exemplo de interação ocorre quando o estudante estiver situado próximo a uma determinada atividade, em que o agente emite um comentário sobre os propósitos e assuntos expostos neste exercício, oferecendo incentivo e apoio em caso de dúvidas.

Além disso, outro recurso incorporado ao ELAI é sua capacidade de adaptação ao contexto do usuário (context-aware computing), por meio de seu nível de expertise, que é capturada conforme informações presentes no ambiente. Essa captura ocorre no momento em que o aluno, por meio de seu avatar, entra no espaço monitorado pelo agente, sendo disparados os scripts para executar uma consulta em uma tabela criada na base de dados do OpenSim, que contém os dados individuais de cada estudante.

Esses dados foram estabelecidos como básico, intermediário e avançado, em que, por meio das informações consultadas na base de dados do OpenSim, o agente ELAI detêm o nível de experiência do usuário. A partir disso, o agente executa uma requisição ao chatterbot desenvolvido junto ao site PandoraBots.

Este chatterbot tem uma base de conhecimento padrão definido na notação AIML e algumas classes incorporadas da ALICE bot ${ }^{1}$. Com o objetivo de fornecer a adaptação personalizada, conforme as preferências dos estudantes, novas informações referentes ao tópico de RC separadas conforme os três níveis de experiência definidos, foram adicionadas a base de conhecimento desenvolvida, uma vez que o formato default (padrão) desta não possui informações específicas desta área (Figura 2 e 3).

\footnotetext{
${ }^{1}$ Disponível em: < http://alice.pandorabots.com/>.
} 


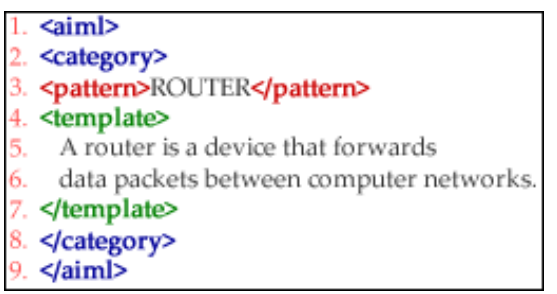

Figura 2. Notação AIML sobre RC

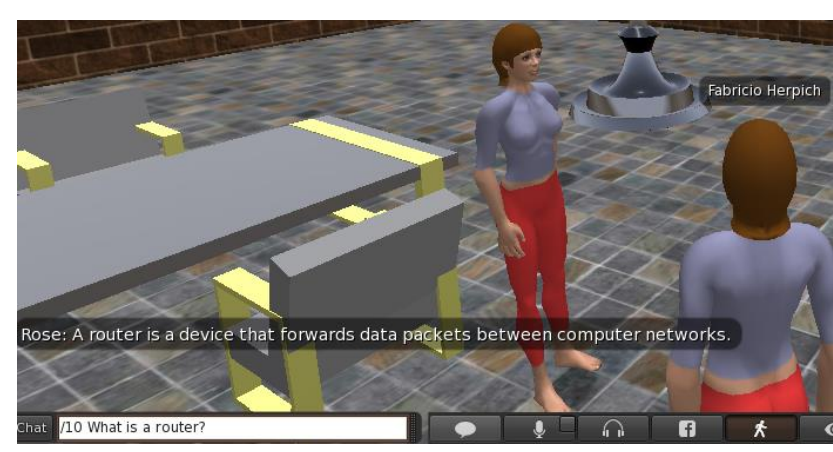

Figura 3. ELAl respondendo sobre roteadores

Com relação aos testes preliminares executados, as Figuras 4, 5 e 6 apresentam três variações de respostas referentes ao tópico questionado (Roteador), para três usuários diferentes, nos quais foram coletadas suas informações de expertise, com o propósito de executar a adaptação das ações do ELAI. Com base nisso, o agente seleciona as respostas apropriadas ao nível de conhecimento indicado dos usuários.

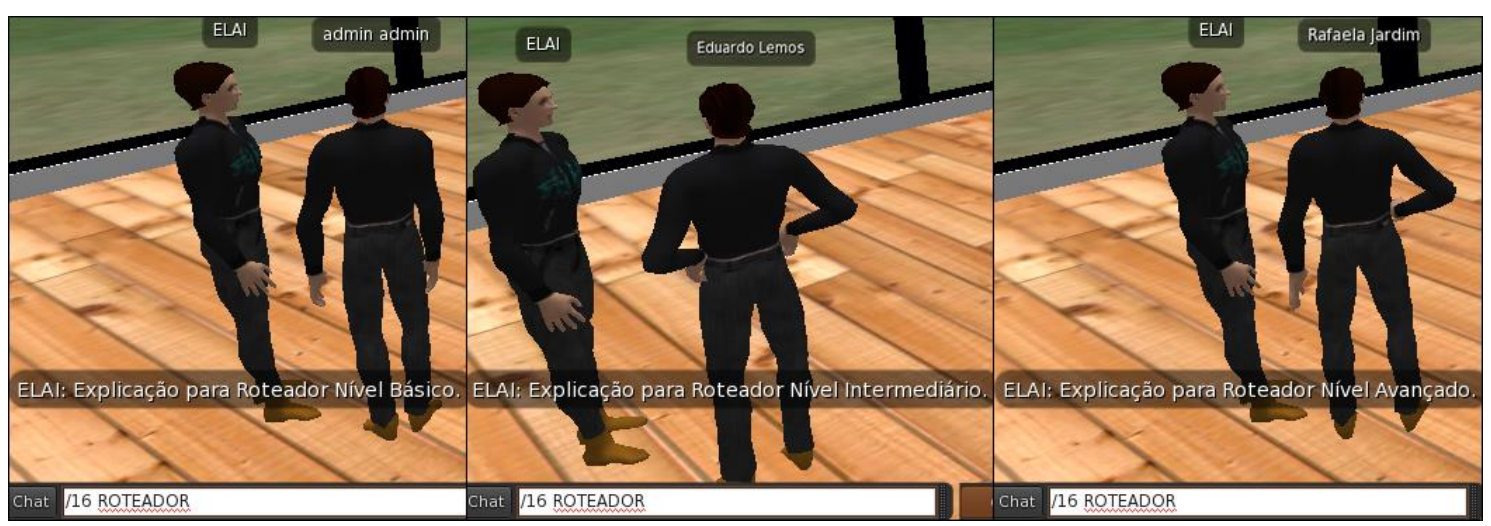

Figura 4. Nível Básico

Figura 5. Nível Intermediário

Figura 6. Nível Avançado

Conforme os recursos implementados no ELAI, uma forma de organizar as atividades executadas pelo educando no MV foi criado um percurso pedagógico no ambiente. Desta forma, a realização das atividades segue uma sequência lógica previamente definida, em que os usuários são monitorados pelo agente com o objetivo de respeitá-la, sendo impossibilitado de tomar vias alternativas a este percurso.

No que diz respeito ao papel do professor, por meio das informações coletadas este poderá avaliar as ações a serem aplicadas com intuito de corrigir e/ou adequar a trajetória para proporcionar melhorias no ensino. Assim, podem ser evidenciadas situações carentes de melhoria, as quais foram identificadas pelos estudantes durante seu processo de aprendizagem, seja por meio da não realização de uma determinada tarefa ou baixas notas, em consequência disso, é possível oferecer uma maior objetividade nos conteúdos e ferramentas para sua educação.

\section{Conclusão e Trabalhos Futuros}

Este artigo apresentou o delineamento de um projeto de pesquisa voltado ao ensino de RC. Para tanto, expõe a implementação de um agente inteligente denominado ELAI, que 
proporciona auxílio aos estudantes provendo um apoio sensível ao contexto que está concentrado no nível de experiência dos mesmos.

Além disso, foram incorporadas outras habilidades ao ELAI com o intuito de ensejar uma maior sensação de imersão pelos estudantes, por meio de funções atribuídas ao agente. Do mesmo modo, os recursos estabelecidos pelo ambiente virtual imersivo $\mathrm{TCN}^{5}$, incorporado a este projeto, possibilitam novas alternativas de aprendizagem aos estudantes, através de simulações de equipamentos e a realização de experimentos, relacionando conteúdos teóricos com a prática.

Diante do provimento de diferentes recursos aos estudantes, foi possível observar como resultado preliminar a potencialidade existente no emprego de agentes inteligentes na educação juntamente aos ambientes virtuais imersivos. Agregado a isso, está o fato destes serem cientes dos contextos de seus usuários, tornando possível a adaptação de conteúdos, materiais e atividades no MV às preferências dos estudantes.

Este estudo propõe como trabalhos futuros a implementação de outros recursos ao agente inteligente ELAI, e.g., aprimorar o seu papel pedagógico no ambiente, fazendo com que consiga instigar os estudantes a resolver desafios. Além disso, pretende-se ampliar a base de conhecimento do chatterbot, desenvolvendo novas classes na notação AIML sobre assuntos relacionados à RC. Também objetiva-se validar sua utilização com estudantes em nível de graduação.

\section{Referências}

Franklin, S. e Graesser, A. (1996) "Is it an Agent, or just a Program?: A taxonomy for Autonomous Agents". In: Proceedings of the Third International Workshop on Agent Theories, Architectures, and Languages, Springer-Verlag.

Medina, R. D. (2004) “ASTERIX: Aprendizagem significativa e tecnologias aplicadas no ensino de redes de computadores: integrando e explorando possibilidades". Tese de Doutorado. Universidade Federal do Rio Grande do Sul, Porto Alegre, 174 páginas.

Piovesan, S. D., Wagner, R., Medina, R. D. e Passerino, L. M. (2013) "Sistema Imersivo para Pessoas com Deficiência". In: XXIV Simpósio Brasileiro de Informática na Educação (SBIE), p. 497-506.

Russell, S. e Nörvig, P. (1995) “Artificial Intelligence: A Modern Approach”. In: Englewood Cliffs, Prentice Hall, Inc. New Jersey, USA.

Silva, T. G. D., Bernardi, G. e Müller, F. M. (2011) “Abordagem de Apoio ao Ensino e Aprendizagem de Teste de Software Baseada em Jogos Sérios e Mundos Virtuais". In: XXII Simpósio Brasileiro de Informática na Educação (SBIE), p. 538-541.

Soliman, M. e Guetl, C. (2010) "Intelligent pedagogical agents in immersive virtual learning environments: A review". In: MIPRO, 2010 Proceedings of the 33rd, p. 827-832.

Voss, G. B. (2014) “TCN5 - Teaching Computer Networks In a Free Immersive Virtual Environment". Dissertação de Mestrado, Universidade Federal de Santa Maria (UFSM), $159 \mathrm{p}$. 